\title{
DIFFERENTIAL EQUATIONS DRIVEN BY ROUGH SIGNALS (I): AN EXTENSION OF AN INEQUALITY OF L. C. YOUNG
}

\section{TERRY LYONS}

A в StRACт. L.C. Young proved that if $x_{t}, y_{t}$ are continuous paths of finite $p, p^{\prime}$ variations in $\mathbb{R}^{d}$ where $\frac{1}{p}+\frac{1}{p^{\prime}}>1$ then the integral $\int_{0}^{t} y_{u} d x_{u}$ can be defined. It follows that if $p=p^{\prime}<2$, and $f$ is vector valued and $\alpha$-Lipschitz function with $\alpha>p-1$, one may consider the non-linear integral equation and the associated differential equation:

$$
\begin{aligned}
y_{t} & =a+\int_{0}^{t} \sum_{i=1}^{d} f^{i}\left(y_{u}\right) d x_{u}^{i} \\
d y_{t} & =\sum_{i=1}^{d} f^{i}\left(y_{t}\right) d x_{t}^{i} \quad y_{0}=a .
\end{aligned}
$$

If one fixes $x$ one may ask about the existence and uniqueness of $y$ with finite $p$-variation where to avoid triviality we assume $d>1$. We prove that if each $f^{i}$ is $(1+\alpha)$-Lipschitz in the sense of [7] then a unique solution exists and that it can be recovered as a limit of Picard iterations; in consequence it varies continuously with $x$. If each $f^{i}$ is $\alpha$-Lipschitz, one still has existence of solutions, but examples of A.M. Davie show that they are not, in general, unique.

\section{Introduction}

This paper is primarily concerned with iterating integrals and with the convergence of Picard's iteration scheme.

By means of a combinatorial inequality similar to one in Young's paper [9], but harder to prove, we show by induction, that if $x$ is continuous and has finite $p$-variation $\rho(t)$ over $[0, t]$, then

$$
x_{t}^{i}=\int_{0<u_{1}<\cdots<u_{n}} \ldots \int_{u_{1}} d x_{u_{1}}^{i_{1}} \ldots d x_{u_{n}}^{i_{n}}, \quad \underline{i}=\left(i_{1}, \ldots, i_{n}\right),
$$

Received April 22, 1994. 
then $x^{\underline{i}}$ has $p$-variation over an interval $[s, t]$ controlled by

$$
[1+\zeta(2 / p)]^{n}\left[\left(\rho(t)^{n}-\rho(s)^{n}\right) / n !\right]^{1 / p},
$$

where $\zeta$ is the Riemann zeta function.

To show that Picard's algorithm converges it seems crucial to express $f(x)-f(y)=(x-y) \cdot g(x, y)$, for in this case, putting

$$
z_{n}(t)=a+\int_{0}^{t} f\left(z_{n-1}\right) d x
$$

and $y_{n}=z_{n}-z_{n-1}$; one has the quasi-linear expression

$$
y_{n+1}(t)=\int_{0}^{t} y_{n}(u) g\left(z_{n}, z_{n-1}\right) d x_{u} .
$$

Providing $g$ is $\alpha$-Lipschitz with $\alpha>p-1$ a priori bounds on $z_{n}$ permits a proof that $\sum_{0}^{\infty} y_{n}$ converges in the p-variation norm over any finite time interval. It follows that if $f$ is $(1+\alpha)$-Lipschitz [7] then the solution to (1) exists for all time.

This note only treats the case where $x$ has finite $p$-variation and $p<2$. In [5] the author outlines an approach to the general case where the path $x$ is assumed to have finite $p<n$ for each $n$. Although the formulation is more complicated one still has continuity and uniqueness results.

\section{Structure of the paper}

In the first part of this note we prove a simple combinatorial lemma.

In the second part, this combinatorial lemma is used to estimate iterated integrals. This section refines results of L. C. Young.

In the third part, the estimates on iterated integrals are exploited to demonstrate that Picard iteration converges and to obtain explicit estimates for the time interval on which this convergence occurs. An extra degree of smoothness is required of $f$ to proceed from the existence results to unique solutions and convergence for Picard's iteration.

\section{A combinatorial lemma}

Suppose $\mathcal{D}$ is a dissection of $[a, b]$ so that $a=r_{0}<r_{1}<\cdots<r_{k}=b$. It will be convenient to introduce difference operators $\delta_{+}^{i}$ and $\delta_{-}^{i}$ for each $i, 0<i<k$. Let $\delta_{+}^{i} f=f\left(r_{i+1}\right)-f\left(r_{i}\right), \quad \delta_{+}^{i} r=r_{i+1}-r_{i}$ and $\delta_{-}^{i} f=$ $f\left(r_{i}\right)-f\left(r_{i-1}\right), \quad \delta_{-}^{i} r=r_{i}-r_{i-1}$ (here $f$ is a function on $[a, b]$ and $r$ a function on $\{0, \ldots, k\})$. 
Suppose $f$ is an increasing non-negative function on $[a, b]$. Let $U^{i}$ be the rectangle $\left[r_{i}, r_{i+1}\right] \times\left[f\left(r_{i-1}\right), f\left(r_{i}\right)\right]$ with area $\left|U_{i}\right|=\delta_{-}^{i} f \delta_{+}^{i} r$. Then the $U^{i}$ form a chain of touching rectangles lying under the graph of $f$ and touching it at the points $\left(r_{i}, f\left(r_{i}\right)\right)$. Our combinatorial result provides, for appropriate $f$, that at least one of these rectangles must have small area. Clearly $\sum_{i=1}^{k-1}\left|U_{i}\right|<\int_{a}^{b} f(u) d u$ and so there exists $i$ with $\left|U_{i}\right| \leq \frac{1}{(k-1)} \int_{a}^{b} f(u) d u$. However with $(k-1)(k-2) / 2$ rectangles $\left[r_{i}, r_{i+1}\right] \times\left[\left(f\left(r_{i^{\prime}}\right), f\left(r_{i^{\prime}+1}\right)\right)\right], i^{\prime}<i$ under $f$ maybe one could prove that

$$
\min _{i}\left|U_{i}\right| \leq \frac{C}{(k-1)^{2}} \int_{a}^{b} f(u) d u \text {. }
$$

We will see in Theorem 1.1 that if $f(s)=s^{N}, N \geq 1, a \geq 0$ then this is true with $C=1$. In retrospect, the independence from $N$ seems a little surprising; the estimate is not true for all increasing $f$ as the following example shows:

Define $r_{0}=0, r_{j}=r_{j-1}+\frac{1}{j !}$, and define $f$ on $\left(r_{0}, r_{1}\right)$ to be $f_{0}=0$, and on $\left[r_{j}, r_{j+1}\right]$ to be $f_{j}=f_{j-1}+j$ !. Then

$$
\int_{r_{0}}^{r_{k}} f d u=\sum_{j=1}^{k-1}(j !)^{-1}(j !+(j-1) !+\cdots+2)
$$

and the right hand side grows linearly in $k$ while each $U_{i}$ has area 1 proving that the original inequality can be sharp.

A simple positive result follows from the geometric/arithmetic mean inequality:

Lemma. There is an $i \in(1, \ldots, k-1)$ such that

$$
\delta_{-}^{i} f \delta_{+}^{i} r \leq \frac{1}{(k-1)^{2}}\left(f\left(r_{k}\right)-f\left(r_{0}\right)\right)\left(r_{k}-r_{0}\right) .
$$

Proof. Let $\lambda=\inf _{i} \delta_{-}^{i} f \delta_{+}^{i} r$; then

$$
\begin{aligned}
\lambda & \leq\left(\left(\prod_{i=1}^{k-1} \delta_{-}^{i} f\right)\left(\prod_{i=1}^{k-1} \delta_{+}^{i} r\right)\right)^{1 / k-1} \\
& \leq\left(\frac{1}{k-1} \sum_{i=1}^{k-1} \delta_{-}^{i} f\right)\left(\frac{1}{k-1} \sum_{i=1}^{k-1} \delta_{+}^{i} r\right) \\
& =\frac{1}{(k-1)^{2}}\left(f\left(r_{k-1}\right)-f\left(r_{0}\right)\right)\left(r_{k}-r_{1}\right) \\
& \leq \frac{1}{(k-1)^{2}}\left(f\left(r_{k}\right)-f\left(r_{0}\right)\right)\left(r_{k}-r_{0}\right) .
\end{aligned}
$$

The main result in this section is the following. 
Theorem 1.1. Suppose $f$ is convex and increasing. Let $\mathcal{D}=\left\{a=r_{0}<\right.$ $\left.r_{1}<\cdots<r_{k}=b\right\}$ be a partition of $[a, b]$; then there is an $i$ such that

$$
\delta_{-}^{i} f \delta_{+}^{i} r \leq \frac{1}{4(k-1)^{2}}\left(\int_{a}^{b}\left|f^{\prime}(u)\right|^{1 / 2} d u\right)^{2} .
$$

In the special case where $f(r)=r^{N}, N \geq 1$, and $a>0$, one obtains the further inequality

$$
\begin{aligned}
\delta_{-}^{i} f \delta_{+}^{i} r & \leq \frac{1}{(k-1)^{2}} \int_{a}^{b} f(u) d u \\
& =\frac{1}{(k-1)^{2}}\left(\frac{b^{N+1}-a^{N+1}}{N+1}\right) .
\end{aligned}
$$

Proof. Start by observing that

$$
\min _{i} \delta_{-}^{i} f \delta_{+}^{i} r \leq\left[\frac{1}{k-1} \sum_{j=1}^{k-1}\left(\delta_{-}^{j} f\right)^{1 / 2}\left(\delta_{+}^{j} r\right)^{1 / 2}\right]^{2} .
$$

Now observe that $\delta f \cong\left|\frac{d f}{d x}\right|^{1 / 2} \delta x\left|\frac{d f}{d x}\right|^{1 / 2}$ and for convex $f$ we have the more precise inequality

$$
f(s)-f(r) \leq \int_{r}^{s}\left(f^{\prime}(u)\right)^{1 / 2} d u\left(f^{\prime}(s)\right)^{1 / 2}
$$

since for increasing positive $h$ one has $\int_{t}^{s} h^{2} \leq h(s) \int_{t}^{s} h$. Further exploiting the monotonicity of $f^{\prime}$ one has

$$
\begin{aligned}
& \min _{i} \delta_{-}^{i} f \delta_{+}^{i} r \leq \\
& \frac{1}{(k-1)^{2}}\left(\sum_{j=1}^{k-1}\left(\left(\int_{r_{j-1}}^{r_{j}}\left(f^{\prime}(u)\right)^{1 / 2} d u\right)\left(\int_{r_{j}}^{r_{j+1}}\left(f^{\prime}(u)\right)^{1 / 2} d u\right)\right)^{1 / 2}\right)^{2} .
\end{aligned}
$$

Now $|c|+|d| \geq 2|c d|^{1 / 2}$ and hence the right hand side of the expression is at most

$$
\frac{1}{4(k-1)^{2}}\left(\int_{a}^{b}\left|f^{\prime}(u)\right|^{1 / 2} d u\right)^{2}
$$

establishing the main part of the result. 
Substituting in for $f(u)$ the power $u^{N}$ yields an upper bound of

$$
\frac{1}{(k-1)^{2}} \frac{N}{(N+1)^{2}}\left(b^{(N+1) / 2}-a^{(N+1) / 2}\right)^{2}
$$

and since $b>a>0$ forces $b^{2}-a^{2}>(b-a)^{2}$ we have the final conclusion

$$
\begin{gathered}
\min _{i} \delta_{-}^{i} r^{N} \delta_{+}^{i} \leq \frac{1}{(k-1)^{2}} \frac{N}{(N+1)}\left(\frac{b^{N+1}-a^{N+1}}{N+1}\right) \\
=\frac{1}{(k-1)^{2}} \frac{N}{N+1} \int_{a}^{b} u^{N} d u .
\end{gathered}
$$

As $N /(N+1)$ is less than one this completes the proof.

One might ask if $\left(\int\left|f^{\prime}(u)\right|^{1 / 2} d u\right)^{2}$ is often comparable to area under the graph of $f$. In general this is not true as one may verify with $e^{\epsilon u}$.

\section{Controlling Iterated Integrals}

Following Wiener, if $p \geq 1$, then we define the $p$-variation of a path $f$ over an interval $J$ as

$$
\omega_{J, p}(f)=\sup _{r_{0}<r_{1}<\cdots<r_{k} \in J} \sum_{j=1}^{k}\left|f\left(r_{j}\right)-f\left(r_{j-1}\right)\right|^{p}
$$

and let $\|f\|_{J, p}=\left|\omega_{J, p}(f)\right|^{1 / p}$. Then $\|\cdot\|_{J, p}$ is a Banach semi-norm and in particular, satisfies the triangle inequality. Any path $f$ with $\|f\|_{J, p}<\infty$ has left and right limits at every point $u$ in $J$, if $\|f\|_{J, p}=0$ then $f$ is a constant.

From the perspective of integration, an important subspace of the functions of finite $p$-variation will be those regular paths for which

$$
\inf _{\epsilon>0} \sup _{\substack{r_{0}<r_{1}<\cdots<r_{k} \in J \\\left|r_{i}-r_{i+1}\right|<\epsilon}} \sum_{j=1}^{k}\left|f\left(r_{j}\right)-f\left(r_{j-1}\right)\right|^{p}=0 .
$$

Any continuous path of finite $p$-variation is of regular finite $p^{\prime}$-variation for any $p^{\prime}>p$.

Let $\zeta(x)$ denote the zeta function (i.e. $\left.\zeta(x)=\sum_{1}^{\infty} \frac{1}{n^{x}}\right)$. 
Suppose the paths $f_{t}$ and $g_{t}$ have finite $p, p^{\prime}$ variation respectively where $1 / p+1 / p^{\prime}>1$. Then an elegant argument by Young shows that, for any Riemann sum one has

$$
\begin{aligned}
\mid g\left(r_{0}\right)\left(f\left(r_{k}\right)-f\left(r_{0}\right)\right)-\sum_{j=0}^{k-1} g\left(r_{j}\right)( & \left.f\left(r_{j+1}\right)-f\left(r_{j}\right)\right) \mid \\
& \leq\left(\zeta\left(1 / p+1 / p^{\prime}\right)\|g\|_{J, p^{\prime}}\right)\|f\|_{J, p} .
\end{aligned}
$$

If one of the paths is regular, the sequence of approximations obtained by refining the partition is easily seen to be Cauchy providing the mesh size converges to zero, and this limit is taken as the definition for the integral. It follows that

$$
\left|\int_{s}^{t} g d f\right| \leq\left(\zeta\left(1 / p+1 / p^{\prime}\right)\|g\|_{J, p^{\prime}}+|g(s)|\right)\|f\|_{J, p}
$$

A simple approximation argument extends the definition of the integral and the inequality to the case where the paths are of finite $p, p^{\prime}$ variation respectively and one of the paths is continuous.

If we view $\int_{0}^{\cdot} g d f$ as a new path we could also ask about its $p$-variation.

$$
\begin{aligned}
& {\left[\sum_{i=1}^{k}\left|\int_{r_{i-1}}^{r_{i}} g d f\right|^{p}\right]^{1 / p} \leq } \\
&\left(\sum_{i=1}^{k}\left|\zeta\left(1 / p+1 / p^{\prime}\right)\|g\|_{\left[r_{i-1}, r_{i}\right], p^{\prime}}\|f\|_{\left[r_{i-1}, r_{i}\right], p}\right|^{p}\right)^{1 / p} \\
&+\left(\sum_{i=1}^{k}\left|g\left(r_{i}\right)\right|^{p}\|f\|_{\left[r_{i-1}, r_{i}\right], p}^{p}\right)^{1 / p}
\end{aligned}
$$

From here there are a number of ways to estimate the $p$-variation of the indefinite integral. Maybe the simplest is that

$$
\left\|\int_{0}^{\cdot} g d f\right\|_{J, p} \leq \zeta\left(1 / p+1 / p^{\prime}\right)\|g\|_{J, p^{\prime}}\|f\|_{J, p}+\|g\|_{\infty}\|f\|_{J, p}
$$

Our intention is to examine the $p$-variation of the integral more carefully.

Theorem 2.1. Suppose $f$ is continuous, $\rho(t)$ is a continuous increasing function of $t$, and that $\|f\|_{[s, t], p} \leq|\rho(t)-\rho(s)|^{1 / p}$ for all $s, t(\rho(s)=$ 
$\omega_{[0, s], p}(f)$ will do). Suppose further that $g(0)=0$, and $\|g\|_{[s, t], p} \leq \mid \rho^{N}(t)-$ $\left.\rho^{N}(s)\right|^{1 / p}$, where $N \geq 1$. Then putting $h(t)=\int_{0}^{t} g d f$ one has

$$
\begin{aligned}
\|h\|_{[s, t], p} & \leq(1+\zeta(2 / p))\left[\frac{\left(\rho^{(N+1) / 2}(t)-\rho^{(N+1) / 2}(s)\right)^{2}}{N+1}\right]^{1 / p} \\
& \leq(1+\zeta(2 / p))\left[\frac{\rho^{N+1}(t)-\rho^{N+1}(s)}{N+1}\right]^{1 / p} .
\end{aligned}
$$

As an immediate corollary one has the following result.

Theorem 2.2. Let $\left(x^{i}\right)_{i=1}^{\infty}$ be paths with $\left\|x^{i}\right\|_{[s, t], p} \leq(\rho(t)-\rho(s))^{1 / p}$. Then the $n^{\text {th }}$ iterated integral thought of as a function of $t$ is of finite p-variation and

$$
\begin{aligned}
\| \int_{0<u_{1}<u_{2}<\cdots<u_{n}=t} \ldots \int_{u_{1}} d x_{u_{2}}^{2} \ldots d x_{u_{n}}^{n} & \|_{[0, T], p} \\
& \leq(1+\zeta(2 / p))^{n-1}\left(\frac{\rho^{n}(T)}{n !}\right)^{1 / p}
\end{aligned}
$$

Proof of Theorem 2.1. We combine Young's approach with the combinatorial lemma. Let $\mathcal{D}$ be some dissection $\left\{s=r_{0}<\cdots<r_{k}=t\right\}$ and let $\int_{\mathcal{D}} g d f$ denote the approximate integral $\sum_{j=0}^{k-1} g\left(r_{j}\right) \delta_{+}^{j}(f)$. Choose $\mathcal{D}$ so that

$$
\left|\left(\int_{\mathcal{D}} g d f-\int_{s}^{t} g d f\right)\right|<\epsilon .
$$

Now let $\mathcal{D}^{i}$ denote the partition obtained by removing $r_{i}$ from $\mathcal{D}$, then

$$
\int_{\mathcal{D}} g d f-\int_{\mathcal{D}^{i}} g d f=\delta_{-}^{i} g \delta_{+}^{i} f \leq\left(\delta_{-}^{i} \rho^{N} \delta_{+}^{i} \rho\right)^{1 / p} .
$$

By the earlier result we can choose $i$ so that this is less than

$$
\left(\frac{1}{(k-1)^{2}}\left(\frac{\rho^{N+1}(t)-\rho^{N+1}(s)}{N+1}\right)\right)^{1 / p} .
$$

Deleting further points one obtains

$$
\begin{aligned}
\left|\left(\int_{\mathcal{D}} g d f\right)\right| \leq \sum_{k=2}^{\infty} \frac{1}{(k-1)^{2 / p}}\left[\left(\frac{\rho^{N+1}(t)-\rho^{N+1}(s)}{N+1}\right)\right]^{1 / p} \\
+|g(s)(f(t)-f(s))|+\epsilon .
\end{aligned}
$$


Now the $p$ th power of the penultimate term in the previous expression is dominated by $\rho(s)^{N}(\rho(t)-\rho(s))$ and this is less than

$$
\frac{\rho^{N+1}(t)-\rho^{N+1}(s)}{N+1}
$$

and so we have (letting $\epsilon \rightarrow 0$ ) that

$$
\left|\int_{s}^{t} g d f\right| \leq\left(1+\zeta\left(\frac{2}{p}\right)\right)\left(\frac{\rho^{N+1}(t)-\rho^{N+1}(s)}{N+1}\right)^{1 / p} .
$$

Using this inequality on sub-intervals we have

$$
\left\|\int_{0}^{t} \mathrm{gdf}\right\|_{[s, t], p} \leq\left(1+\zeta\left(\frac{2}{p}\right)\right)\left(\frac{\rho^{N+1}(t)-\rho^{N+1}(s)}{N+1}\right)^{1 / p} .
$$

which settles theorem 2.1.

Theorem 2.2. follows immediately using the obvious induction.

\section{On the convergence of Picard iteration}

Suppose $x$ is a continuous path in $\mathbb{R}^{d}$ with finite $p$-variation where $p<2$ and let $\left(f^{i}\right)_{i=1}^{d}$ be differentiable vector fields on a space $W$. Suppose further that $W$ is a vector space and the $f^{i}$ are $\alpha$-Lipschitz where $\alpha>p-1$.

Given a path $z_{0}$ in $W$ of finite $p$-variation one may form a second path $z_{1}$ also of finite $p$-variation by

$$
P_{f} z_{0}(t):=z_{1}(t)=a+\int_{0}^{t} f\left(z_{0}(u)\right) d x_{u} .
$$

We say we perform Picard iteration if we continue the process:

$$
z_{n}(t)=a+\int_{0}^{t} f\left(z_{n-1}(u)\right) d x_{u}
$$

We say that $z_{0}$ is a solution to the equation

$$
d z_{0}(t)=\sum_{i=1}^{d} f^{i}\left(z_{0}(t)\right) d x_{t}^{i}
$$

if and only if $z_{1}=z_{0}$, or equivalently if $z_{0}$ is a fixed point of $P_{f}$. 
From the bounds mentioned earlier, and using fixed point theorems it is easy to see that the equation will have solutions under these hypotheses. However, they will not in general be unique or constructively approximated.

This section is primarily concerned with the question: When does the sequence $P_{f}^{n} z$ of Picard iterates converge? We will see that this happens naturally when $f$ has one additional derivative (e.g. $f$ is in the Lipschitz space $\Lambda_{\alpha+1}$ ). In so doing we establish uniqueness results. One motivation for getting $1 / n$ ! into our previous analysis of the iterated integrals is to enable us to prove that Picard iteration converges over the whole positive time interval. (See [2], [6] for stochastic counterparts).

\section{Existence of solutions.}

Let us first estimate the growth of the $p$-variation of $z_{n}$ for $f$ in $\Lambda_{\alpha}$ with $\alpha>p-1$. Suppose

$$
z_{n+1}(t)=a+\int_{0}^{t} f\left(z_{n}\right) d x
$$

then an application of Young's basic inequality shows that

$$
\left\|z_{n+1}\right\|_{[0, t], p} \leq\left(\zeta((1+\alpha) / p)\left\|f\left(z_{n}\right)\right\|_{[0, t], p / \alpha}+\|f(z)\|_{\infty}\right)\|x\|_{[0, t], p}
$$

or slightly more precisely

$$
\leq\left((1+\zeta((1+\alpha) / p))\|f\|_{\Lambda_{\alpha}}\left\|z_{n}\right\|_{[0, t], p}^{\alpha}+|f(a)|\right)\|x\|_{[0, t], p} .
$$

From this bound we will easily see that the operator $P_{f}$ has fixed points. We consider the Banach space of paths with initial point $a$ and finite $p$ variation.

Lemma. There exist solutions $z$ to equation $(1)$ on $[0, t]$ with finite $p^{\prime}$ variations for any $p^{\prime} \in(p, 2)$. Moreover any such solution must lie in the ball

$\|z\|_{[0, t], p}<2|f(y)|\|x\|_{[0, t], p}+\left(2(1+\zeta((1+\alpha) / p))\|f\|_{\Lambda_{\alpha}}\|x\|_{[0, t], p}\right)^{1 /(1-\alpha)}$.

Proof. Put $\theta=\zeta((1+\alpha) / p)\|f\|_{\Lambda_{\alpha}}$ and $\lambda=|f(a)|$. Choose $t$ and $R$ so that $\left(\theta / R^{1-\alpha}+\lambda / R\right)\|x\|_{[0, t], p}<1$. By the remarks above the lemma we have $\left\|z_{n+1}\right\|_{[0, t], p} \leq\left(\theta\left\|z_{n}\right\|^{\alpha}+\lambda\right)\|x\|_{[0, t], p}$ and hence for $\left\|z_{n}\right\|_{[o, t], p}>R$ one has $\left\|z_{n+1}\right\|_{[o, t], p}<\left\|z_{n}\right\|_{[o, t], p}$. Hence any fixed point must occur inside the closed ball of radius $\mathrm{R}$. On the other hand $P_{f}$ maps the closed ball of radius $R$ in the $p$-variation paths to itself. It follows that it maps its (compact) closure in the space of $p^{\prime}$-variation paths to itself. As this set is convex $P_{f}$ must have a fixed point by Tychonoff's fixed point theorem[8]. Hence solutions exist for any $p^{\prime} \in(p, 2)$.

Remark. It is immediate that, providing that $x$ is continuous, solutions to (1) with locally finite $p^{\prime}$ variation exist for all time. 
Convergence of Picard iteration and uniqueness of solution.

A crucial point in the proof of convergence of Picard iteration is the following standard lemma.

Lemma. Let $f$ be in the Lipschitz space $\Lambda_{\beta}, \beta>1$; then there exists in $\Lambda_{\beta-1}$ a Lipschitz function $g: W \times W \rightarrow W^{*}$ such that

$$
f(x)-f(y)=\sum_{j=1}^{d}(x-y)^{j} g_{j}(x, y) .
$$

Moreover, the norms of $f$ and $g$ are equivalent if $\|f\|_{\infty}$ is finite.

Proof. The analogous result where $f$ is $C^{n}$ is routine; the essential lemma required to modify the argument for the Lipschitz case is Proposition 9 on p.147 of [7].

The significance of the lemma lies in the way it allows one to re-express Picard iteration as a quasi-linear procedure, and it is this rather than any contraction mapping principle that ensures the convergence of the scheme. Let $z_{n}$ be defined as before, but now let $y_{n}=z_{n}-z_{n-1}$. Thus $y_{1}=z_{1}-z_{0}$, and

$$
y_{n+1}=\int_{0}^{t} y_{n}(u) g\left(z_{n}(u), z_{n-1}(u)\right) d x_{u} .
$$

We will prove that the $y_{n}$ decay to zero sufficiently rapidly to obtain convergence of the $z_{n}$.

\section{An a priori bound.}

Suppose $f$ is bounded, $\rho$ is continuous and increasing function, and that for all $s, t$

$$
\|x\|_{|s, t|, p} \leq|\rho(s)-\rho(t)|^{1 / p}, \quad\left\|z_{0}\right\|_{|s, t|, p} \leq|\rho(s)-\rho(t)|^{1 / p}
$$

$\left(z_{0}=a\right.$ will do $)$.

If $f$ is in $\Lambda_{\beta}$ with $2>\beta>1$, then $f$ has a continuous bounded derivative, and using previous estimates one has that

$$
\left\|z_{n}\right\|_{[s, t], p} \leq\left(\zeta(2 / p)\|\nabla f\|_{\infty}\left\|z_{n-1}\right\|_{[s, t], p}+\|f\|_{\infty}\right)(\rho(t)-\rho(s))^{1 / p} .
$$

So that providing $|\rho(t)-\rho(s)|^{1 / p}<\frac{1-\epsilon}{\zeta(2 / p)\|\nabla f\|_{\infty}}$, the sequence $\left\|z_{n}\right\|_{[s, t], p}$ is uniformly less than $\left(1+\|f\|_{\infty} / \epsilon\right)[\rho(t)-\rho(s)]^{1 / p}$ for all $n$. Taking $\epsilon=1 / 2$ it follows that

$$
\left\|z_{n}\right\|_{[s, t], p} \leq\left(1+2\|f\|_{\infty}\right)[\rho(t)-\rho(s)]^{1 / p}+\left(2 \zeta(2 / p)\|\nabla f\|_{\infty}\right)[\rho(t)-\rho(s)]
$$


holds uniformly for all $s, t$ and $\mathrm{n}$. Changing constants slightly we have essentially the same estimate for $\left\|g\left(z_{n}, z_{n-1}\right)\right\|_{[s, t], p /(\beta-1)}$ because $\|g\|_{\Lambda_{\beta-1}}$ is finite:

$$
\left\|g\left(z_{n}, z_{n-1}\right)\right\|_{[s, t], p /(\beta-1)} \leq A[\rho(t)-\rho(s)]^{(\beta-1) / p}+B[\rho(t)-\rho(s)]^{\beta-1}
$$

uniformly in $s, t$ and $n$.

Let $h_{n}(t)=\int_{0}^{t} g\left(z_{n}, z_{n-1}\right) d x ;$ then

$$
\begin{aligned}
\left\|h_{n}(\cdot)\right\|_{[s, t], p} & =\left\|h_{n}(\cdot)-h_{n}(t)\right\|_{[s, t], p} \\
& \leq\left(\zeta(\beta / p)\|g\|_{[s, t], p /(\beta-1)}+2\|g\|_{\infty}\right)\|x\|_{[s, t], p} \\
& \leq \zeta(\beta / p)\left[A(\rho(t)-\rho(s))^{(\beta-1) / p}+B(\rho(t)-\rho(s))^{(\beta-1)}+2\|g\|_{\infty}\right] \\
& \cdot[\rho(t)-\rho(s)]^{1 / p} .
\end{aligned}
$$

In particular there is some increasing function $A(T)$ so that for all $s, t<T$

$$
\left\|h_{n}(\cdot)\right\|_{[s, t]} \leq A(t)[\rho(t)-\rho(s)]^{1 / p} .
$$

Theorem. For any interval $[0, T]$, and for $f$ in $\Lambda_{\beta}$ where $p<\beta \leq 2$, Picard iteration converges.

Proof. By hypothesis $y_{1}\left(=z_{1}-z_{0}\right)$ satisfies the inequality

$$
\left\|y_{1}\right\|_{[s, t]} \leq L|\rho(t)-\rho(s)|^{1 / p}
$$

on $[0, T]$. Since

$$
y_{n+1}=\int_{0}^{t} y_{n} d h_{n}
$$

it follows from our main theorem that

$$
\left\|y_{N}\right\|_{[s, t], p} \leq[1+\zeta(2 / p)]^{N-1} A(T)^{N} L^{N}\left|\left(\frac{\rho^{N}(t)-\rho^{N}(s)}{N !}\right)\right|^{1 / p}
$$

Because $(N !)^{1 / p}$ dominates any geometric series it follows that the series

$$
\sum_{i}^{\infty} y_{N}
$$

converges in $p$-variation norm on $[0, T]$ for all $T$ proving that Picard iteration converges. 
One would expect a Picard iteration argument to demonstrate uniqueness. Let $z$ represent a solution, then

$$
\left(z_{n+1}-z\right)=\int_{0}^{t}\left(z_{n}-z\right) g\left(z_{n}, z\right) d x
$$

Our a priori estimates are already sufficient to see by the above argument that on any fixed time interval

$$
\left\|z_{n}-z\right\|_{[0, T]}=o\left(\left(\frac{k^{n}}{n !}\right)^{1 / p}\right)
$$

for some large $p$. This confirms that $z$ is indeed unique and that ultimately convergence is very rapid.

Continuity on $[0, T]$ in the $p$-variation norm in $x$ also follows routinely. For the estimates above prove the uniform convergence of $z_{n}$ to $z$ in $p$ variation norm. Since the terms $z_{i}$ are patently continuous in $x$ continuity follows.

\section{Remarks}

This paper gives equation (1) pathwise meaning, and demonstrates the existence, uniqueness and continuity of solutions whenever $x$ is $p$-Lipschitz and $p<2$ and $f$ is suitably smooth. In [5] we treat the complementary, and more complex situation where $p>2$, so we do not discuss it further now.

Although it is quite standard to make an identification between solutions to differential equations and solutions to the associated integral equations, a little reflection should convince the reader that the approach makes an implicit assumption requiring verification. In the equation (1) the path $x$ belongs in a vector space with a prescribed coordinate chart, however each $f^{i}$ is a vector field defined unambiguously without reference to a particular choice of coordinate chart; we therefore expect $y$ to be a path on a manifold and to be independent of the choice of coordinates. However, the representation of $y$ as the solution to an integral equation only makes sense after one has made a choice of coordinate chart. One should therefore look at the independence of the solution under changes in coordinates. Fortunately, it is easy to show that the any of the solutions defined through the Young integrals are invariant under $(1+\alpha)$-Lipschitz changes of coordinates.

Discontinuous $x(t)$.

Consider a Lévy process whose paths almost surely have finite $p$-variation for some $p<2$. For example, the Cauchy process or some other symmetric 
stable process. Let $x$ be a realisation from that process. Can we apply the results above to give pathwise existence and uniqueness of solutions to (1). Our paper has, so far, discussed only continuous driving signals x. This was important as there is no sensible definition for Young's integral for a general pair of functions $x$ and $y$ if both have a jump at the same time. However for our application of solving differential equations the story is different: there is always a sensible choice for the evolution of our solution $y$ over a jump in $x$. Recall that $x$ naturally lives in a vector space, although $y$ lives on a manifold, moreover if we reparameterise a continuous path $x$, then the solution $y$ of the differential equation (1) is the reparameterisation of the original one. So, if at each jump $t_{-}, t$ or $t, t_{+}$in the path $x$ we insert a linear segment and reparameterise the path so that this piece is traversed in a time of order $\left|x_{t_{-}}-x_{t}\right|^{2+\epsilon}$, etc. then the path still has finite $p$ variation. We can now solve the equation as described above and then reparameterise the time variable so as to make the jumps instantaneous again. It is essentially trivial that this procedure gives a unique $y$ which we could reasonably regard as the solution to the equation.

\section{Related results.}

A number of authors $[1,3,9]$ have considered finite $p$-variation paths and integrals - however the nonlinear question of solving differential equations pathwise seems to have escaped consideration. Others, and particularly $[6,2]$ have looked at the question of convergence of Picard's iterates over the whole time axis in stochastic settings. Itô [4] considered SDE's driven by Lévy processes in his original memoir.

Dr A.M. Davie has shown me examples of multiple solutions to (1) when $f$ is $C^{\alpha}, x$ is of finite $p$ variation, and $\alpha<p<2$.

\section{Acknowledgements}

The author freely acknowledges the help and encouragement of his numerous colleagues. Originally this paper was formulated in terms of Hölder continuity not $p$-variation in which case the interval selection trick of Young is replaced by a comparison of sums over diadic partitions. Jean Bertoin suggested the change - it has clarified a number of issues. Dan Stroock also made some helpful presentational remarks. The initial push happened during a pleasant week in Madrid as guest of José Fernandez. The author thanks his student David Williams for identifying many misprints.

\section{References}

1. J. Bertoin, Sur une intégrale pour les processus à $\alpha$-variation bornée, The Annals of Probability 17 (1989), 1521-1535. 
2. K. Bichteler, Stochastic integration and $L^{p}$ theory of semimartingales, The Annals of Probability 9 (1981), 49-89.

3. M. Bruneau, Lecture Notes in Math, vol. 413, Springer, Berlin-New York, 1974.

4. K. Ito, On stochastic differential equations, Memoirs American Math. Soc. 4 (1951).

5. T. J. Lyons, The interpretation and solution of ordinary differential equations driven by rough signals, MSS, to appear in Proceedings of Cornell Meeting on Stochastic Analysis, AMS, 1994 (1993).

6. L. Schwartz, La convergence de la serie de Picard pour les EDS (equations differentielles stochastiques), Collection: Seminaire de Probabilites, vol. XXIII, 1989, pp. 343-354; Lecture Notes in Math., vol. 1372, Springer, Berlin-New York, 1989.

7. E. M. Stein, Singular Integrals and Differentiability Properties of Functions (1970), Princeton University Press, Princeton.

8. A. Tychonoff, Ein Fixpunktsatz, Math. Ann. 111 (1935), 767-776.

9. L. C. Young, An inequality of Hölder type, connected with Stieltjes integration, Acta Math. 67 (1936), 251-282.

Department of Mathematics, Imperial College, London, SW 72 B Z

E-mail address: t.lyons@ic.ac.uk 